\title{
PAEDIATRIC SURGICAL ONCOLOGY IN DEVELOPING COUNTRIES: A PERSPECTIVE FROM PAKISTAN
}

Surgical oncology over the years has established itself as a distinct speciality, with defined goals and curriculum in technologically advanced countries, sadly it has yet to gain recognition in developing countries like Pakistan. Cancer treatment has been a field of extensive research and significant advancements have taken place over the years. This includes surgical management of patients with solid tumours. New treatment protocols and surgical approaches have made a remarkable reduction in mortality and morbidity; however, in context of Pakistan and other developing countries, this change is not noticeable. This has been reported in studies from India and Ethiopia. ${ }^{[1,2]}$ In a report on the subject of global challenges related to cancer in children, it was mentioned that most of the cancers in children are curable and about $80 \%$ of children with cancer in developing world survive while the same number die in developing countries, a painful fact indeed. ${ }^{[3]}$

In Pakistan, paediatric surgical oncology has not received much attention. Adult surgeons still manage majority of paediatric oncology patients suffering from malignancies related to head and neck, ENT, neurosurgery, orthopaedics and ophthalmology. General paediatric surgeons are usually involved in the management of abdominal and thoracic malignancies. These paediatric surgeons are not formally trained. Most of them have learnt the surgical techniques in an apprenticeship model. Thus, they may be good at surgery but are not knowledgeable about the peculiarities and minute surgical details that are part of overall treatment of such patients. It is, therefore, not uncommon to find a poorly investigated patient being operated in whom surgery was not indicated. Such intervention may be counterproductive. Surgery plays an important role in overall treatment of solid tumours. Some of the surgical procedures are also performed for palliation and rehabilitation purposes. Thus, surgeons cannot be accepted to play a role of simply cutting and then handing over the patients to oncologists. Paediatric

Correspondence: Dr. Jamshed Akhtar,

Department of Paediatric Surgery, National Institute of Child Health, Jinnah Sindh Medical University, Karachi, Pakistan.

Email: jamjim88@yahoo.com surgeons are now posted in many district level hospitals in Pakistan; however, to ensure an optimal treatment, they need training and updating on recent advances and treatment protocols.

It is important at this point to trace a history of paediatric surgical oncology in Pakistan and especially in the province of Sindh. Paediatric surgical oncology in Pakistan was started in 1963 in the Department of Paediatric Surgery, which was part of the School of Paediatrics at Jinnah Central Hospital, Karachi. It was due to special interest of the paediatric surgeons, who not only established paediatric surgery as a discipline but also its subspecialities in Pakistan. Paediatric surgeons at that time operated on paediatric patients with solid tumours and also presented their work at international and national conferences. Professor Nizam ul Hassan was most active in this regard. He also wrote number of articles on paediatric surgical oncology. He also presented idea of partial nephrectomy in mid-70s in patient with Wilms' tumour limited to one pole of the kidney. In 1982, chemotherapy drugs became available in Pakistan which paved way to more formal solid tumour management program. The chemotherapy for the paediatric solid tumour patients was started for the $1^{\text {st }}$ time in the National Institute of Child Health (NICH), Karachi. At that time, physicians in general paediatric medical units were used to provide chemotherapy for leukaemia patients and solid tumours patients received chemotherapy in surgical units according to the international protocols. Many innovations were also introduced in the oncology practices at that time. The first FNAC in Pakistan was performed by Professor Qazi Shakoor in Surgical Unit B of NICH on a patient with neuroblastoma.

A need of formal care for paediatric oncology patients resulted in establishment of the first Paediatric Oncology Unit of Pakistan in 1999 at NICH, Karachi, with the support of an NGO, Child Aid Association. This paved the way toward a comprehensive treatment for paediatric cancer patients. Dr. Shamvil Ashraf, a renowned oncologist, remained part of this facility in his early professional career and contributed a lot. He later went 
onto establish Children Cancer Hospital in Karachi which now has become part of Indus Hospital.

The organized approach to paediatric patients with solid tumours cannot be ignored. It is important to develop a close liaison between oncologists, surgeons, other related health-care specialists and nurses. Tumour board also plays an important role. This forum is active in Karachi since 2010. It helped in getting advice from experts who share their experiences at this forum. Pakistani oncologists working in the US, the UK, and Canada are always there to extend support by guiding and helping in planning management of these patients.

It is important for surgeons to follow defined protocols at the time of removal of the tumour and document minute details of the operation. This helps oncologists in staging of tumour and selecting appropriate chemotherapy protocols. Uniform reporting of surgical specimen is also important. Trained histopathologists are key persons in making diagnosis, especially on FNAC samples and core biopsies. The use of panel of the tumour markers, which are now available in Pakistan, greatly facilitates in identification of the type of tumour. There should be no excuse in recent times of not using these services.

Government has provided number of facilities for cancer patients in Pakistan. It has established dedicated hospitals and oncology units at various tertiary care hospitals. NGOs are also very active and provide lot of support in collaboration with philanthropists. Shaukat Khanum Memorial Cancer Hospital and Research Centre played an important role in strengthening cancer-related services in Pakistan, including paediatric surgical oncology.

In treating oncology patients, it is also important to understand the cultural and socioeconomic peculiarities as some of them create hurdles. Like other developing countries, many people believe that cancer is incurable. Most of the patients present late with advanced stage of disease. Many of them are lost to follow-up. Standard chemotherapy protocols, therefore, cannot be provided. ${ }^{[4]}$ Mortality is, therefore, still high. Many patients are brought from faroff places. Travelling long distances and non-availability of accommodation in big cities are important issues to be addressed. These barriers lead to imperfect utilization of services, even when these are offered free of cost at tertiary care centres. Establishing satellite units for providing chemotherapy and intensive care can be a way out.

Research in the field of paediatric oncology cannot be ignored. At present, no formal population-based tumour registry exists in Pakistan. Data from different hospitals are available which are not comprehensive. This is a real challenge as burden of disease cannot be quantified. Pakistan Society of Paediatric Oncology (PSPO) can do a lot in this regard. PSPO is quite active and recently organized its second meeting at Lahore. It has also planned to work on devising protocols in Pakistani context and has made various subgroups to address these issues. To improve paediatric surgical oncology outcome, it is important to educate surgeons, develop liaison, conduct regular multidisciplinary tumour board meetings and organize workshops, conferences and sign MOUs for faculty exchange programs with developed countries. Organizations like the World Health Organization have made a pledge to reduce the burden of non-communicable diseases. Cancer research is one such area and its importance is well recognized. ${ }^{[5]}$ Funding is available for research in this field. Optimal use of such opportunities can go a long way to improve mortality and morbidity of paediatric patients with solid tumours in Pakistan.

\section{Jamshed Akhtar}

Department of Paediatric Surgery, National Institute of Child Health, Jinnah Sindh Medical University, Karachi, Pakistan

Received: 11 June 2017/Accepted: 25 June 2017

\section{References}

1. Agarwala S. Pediatric surgical oncology in India. J Indian Assoc Pediatr Surg 2014;19:187-8.

2. Haileamlak A. The challenge of childhood cancer in developing countries. Ethiop J Health Sci 2016;26:199-200.

3. A Global Challenge: Treating Children with Cancer in Developing Countries. Available from: http://www.healio. com/hematology-oncology/palliative-care/news/print/hemonctoday/\%7B73828ce7-bd54-4570-9ef9-aa35c9389ce9\%7D/aglobal-challenge-treating-children-with-cancer-in-developingcountries. [Last accessed on 2017 Jun 01].

4. Ekenze SO, Agugua-Obianyo NE, Odetunde OA. The challenge of nephroblastoma in a developing country. Ann Oncol 2006; 17:1598-600.

5. Schultz A. The ESMO Developing Countries Task Force Developing Countries Oncology Survey (DC-OS) Report; 2006. Available from: https://www.esmo.org/content/ download/8399/170349/file/DC_Survey_Report_Istanbul. pdf. [Last accessed on 2017 Jun 01]. 